\title{
Combining MRI with NIHSS Thresholds to Predict Outcome in Acute Ischemic Stroke: Value for Patient Selection
}

\author{
P.W. Schaefer, B. Pulli, W.A. Copen, J.A. Hirsch, T. Leslie-Mazwi, L.H. Schwamm, O. Wu, R.G. González, and A.J. Yoo
}

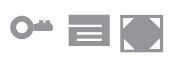

\begin{abstract}
BACKGROUND AND PURPOSE: Selecting acute ischemic stroke patients for reperfusion therapy on the basis of a diffusion-perfusion mismatch has not been uniformly proved to predict a beneficial treatment response. In a prior study, we have shown that combining clinical with MR imaging thresholds can predict clinical outcome with high positive predictive value. In this study, we sought to validate this predictive model in a larger patient cohort and evaluate the effects of reperfusion therapy and stroke side.
\end{abstract}

MATERIALS AND METHODS: One hundred twenty-three consecutive patients with anterior circulation acute ischemic stroke underwent MR imaging within 6 hours of stroke onset. DWI and PWI volumes were measured. Lesion volume and NIHSS score thresholds were used in models predicting good 3-month clinical outcome (mRS 0-2). Patients were stratified by treatment and stroke side.

RESULTS: Receiver operating characteristic analysis demonstrated $95.6 \%$ and $100 \%$ specificity for DWI $>70 \mathrm{~mL}$ and NIHSS score $>20$ to predict poor outcome, and $92.7 \%$ and $91.3 \%$ specificity for PWI (mean transit time) $<50 \mathrm{~mL}$ and NIHSS score $<8$ to predict good outcome. Combining clinical and imaging thresholds led to an $88.8 \%(71 / 80)$ positive predictive value with a $65.0 \%(80 / 123)$ prognostic yield. One hundred percent specific thresholds for DWI (103 versus $31 \mathrm{~mL}$ ) and NIHSS score (20 versus 17) to predict poor outcome were significantly higher in treated (intravenous and/or intra-arterial) versus untreated patients. Prognostic yield was lower in right- versus left-sided strokes for all thresholds (10.4\%-20.7\% versus $16.9 \%-40.0 \%)$. Patients with right-sided strokes had higher 100\% specific DWI (103.1 versus $74.8 \mathrm{~mL})$ thresholds for poor outcome, and the positive predictive value was lower.

CONCLUSIONS: Our predictive model is validated in a much larger patient cohort. Outcome may be predicted in up to two-thirds of patients, and thresholds are affected by stroke side and reperfusion therapy.

ABBREVIATIONS: AUC = area under the curve; IA = intra-arterial; PPV = positive predictive value; $\mathrm{Tmax}=$ time at which the scaled residue function reached its maximum

$\mathbf{R}$ eperfusion therapy improves outcomes of patients with acute ischemic stroke. ${ }^{1,2}$ The decision to treat is primarily determined by the time from symptom onset. Use of IV-rtPA is restricted to the $3-^{1}$ or 4.5 -hour ${ }^{2}$ time window, resulting in only $1 \%-7 \%$ of patients receiving IV-rtPA. ${ }^{3}$ Advanced neuroimaging provides information about a patient's physiology that may be useful to guide treatment

Received June 27, 2014; accepted after revision July 21.

From the Departments of Radiology (P.W.S., B.P., W.A.C., J.A.H., T.L.-M., O.W., R.G.G., A.J.Y.) and Neurology (L.H.S.), Massachusetts General Hospital, Harvard Medical School, Boston, Massachusetts.

P.W. Schaefer and B. Pulli contributed equally to this work.

Please address correspondence to Pamela W. Schaefer, MD, Department of Radiology, Neuroradiology Division, Massachusetts General Hospital, 55 Fruit St, Boston, MA 02114; e-mail: pschaefer@partners.org

- Indicates open access to non-subscribers at www.ajnr.org

三 Indicates article with supplemental on-line table.

Indicates article with supplemental on-line photo.

http://dx.doi.org/10.3174/ajnr.A4103

decisions, especially in an extended time window. Selecting patients for reperfusion therapy on the basis of the mismatch between lesions in diffusion-weighted and perfusion images has been proposed, ${ }^{4}$ but this approach has not been uniformly proved to predict a beneficial treatment response. ${ }^{5-8}$ On the basis of findings that patients with DWI infarct volumes of $>70 \mathrm{~mL}$ have poor outcome regardless of treatment ${ }^{9,10}$ and that the NIHSS score is a strong predictor of outcome, ${ }^{11}$ we recently published a predictive model that combined DWI and mean transit time lesion volumes with NIHSS score thresholds to predict outcome in patients with anterior circulation acute ischemic stroke. ${ }^{12}$ DWI lesion volume $>72 \mathrm{~mL}$ or NIHSS score $>20$ predicted poor outcome (mRS, 3-6), while MTT lesion volume $<47 \mathrm{~mL}$ or NIHSS score $<8$ predicted good outcome (mRS, $0-2$ ) with a high positive predictive value (PPV) when used in combination, in two-thirds of patients.

In this study, we sought to validate our predictive model in a larger, independent cohort of patients and investigated the effects of reperfusion therapy and side of involvement on these thresholds. 


\section{MATERIALS AND METHODS}

\section{Patient Selection and Assessment}

We retrospectively examined the clinical and imaging data of consecutive patients with acute ischemic stroke admitted to our comprehensive stroke center. Inclusion criteria were the following: 1) DWI and PWI obtained within 6 hours of the patient being symptom-free, 2) acute MCA stroke, 3) NIHSS score $>2$, and 4) sufficient clinical follow-up to determine the mRS score at 90 days. This study was approved by our institutional review board. Records were maintained in compliance with the Health Insurance Portability and Accountability Act.

We identified 198 patients with acute ischemic stroke who underwent DWI and PWI within 6 hours. Seventy-five were excluded because of the lack of a follow-up mRS score $(n=28)$, posterior circulation infarction $(n=29)$, calculation of perfusion maps with a delay-sensitive deconvolution technique $(n=9)$, or image quality insufficient for analysis $(n=9)$ ). The remaining 123 patients were included for further analysis. This cohort was independent of the previously published cohort, ${ }^{12}$ without overlap. In patients who received IV-tPA, treatment was started within 4.5 hours. In patients who received intra-arterial (IA) therapy, treatment was initiated within 8 hours after symptom onset.

Admission NIHSS score was determined by a stroke neurologist. Clinical outcome was evaluated by using the mRS score 90 days after stroke onset, which was determined by a stroke neurologist in 59 instances (48\%) and was retrieved from a neurologic examination in the patient's medical record for the remaining patients. Patient outcomes were dichotomized into good (mRS $0-2)$ versus poor ( $\mathrm{mRS} 3-6)$.

\section{Image Acquisition, Postprocessing, and Analysis}

MR imaging was performed on a 1.5T Signa scanner (GE Healthcare, Milwaukee, Wisconsin). DWI was performed with a singleshot echo-planar spin-echo sequence with two $180^{\circ}$ radiofrequency pulses. Three images per section were acquired at $b=0$ $\mathrm{s} / \mathrm{mm}^{2}$, followed by 25 at $b=1000 \mathrm{~s} / \mathrm{mm}^{2}$. Imaging parameters were TR/TE, 5000/80-110 ms; FOV, 22-cm; matrix, $128 \times 128$ zero-filled to $256 \times 256$; 5-mm thickness; 1 -mm gap.

PWI was performed by using a dynamic-susceptibility technique. Serial echo-planar gradient-echo images were acquired with TR/TE, 1500/40 ms; FOV, 22 cm; matrix, $128 \times 128$; section thickness, 5 mm with a $1-\mathrm{mm}$ gap. Twenty milliliters of gadopentetate dimeglumine, $0.5 \mathrm{mmol} / \mathrm{mL}$ (Bayer HealthCare Pharmaceuticals, Wayne, New Jersey), was injected intravenously at $5 \mathrm{~mL} / \mathrm{s}$, beginning 10 seconds after scanning started, followed by $20 \mathrm{~mL}$ of normal saline.

PWI data were processed by using locally written, automated software. Signal-versus-time curves for each pixel were converted to $\Delta$-R2-versus-time curves. $\mathrm{CBV}$ was calculated by integrating the area under these curves; $\mathrm{CBF}$, as the amplitude of the scaled residue function yielded by delay-insensitive singular-value deconvolution $^{13}$; Tmax, as the time at which the scaled residue function reached its maximum; and MTT, as CBV/CBF.

For quantitative measurement, visually detected DWI and MTT abnormalities were segmented in randomized order by 2 experienced neuroradiologists blinded to clinical information by using a semiautomated commercial analysis program (Analyze, Version 7.0; (AnalyzeDirect, Overland Park, Kansas). Tmax maps were thresholded to 6 seconds. Absolute mismatch was defined as MTT - DWI; percentage mismatch, as (MTT - DWI) / DWI $\times$ $100 \%$.

\section{Statistical Analysis}

Statistical analysis was performed by using MedCalc for Windows, Version 11.2.1 (MedCalc Software, Mariakerke, Belgium). $P$ values $<.05$ were considered significant. Clinical and imaging data were compared by using the Student $t$ test or Mann-Whitney $U$ test, and categoric data, by using the $\chi^{2}$ test. Receiver operating characteristics curves were calculated for DWI, MTT, Tmax, and the NIHSS score relative to clinical outcome. Areas under the curve (AUCs) were compared and stratified by reperfusion therapy and stroke side. Prognostic yield was defined as the proportion of patients fulfilling at least 1 threshold criterion.

\section{RESULTS}

\section{Baseline Clinical and Imaging Characteristics, Treatment, Outcomes, and Predictors of 3-Month mRS}

Sixty-eight patients $(55.3 \%)$ had good clinical outcome 90 days after stroke onset (Table 1). There were no significant differences in age, sex, right-hemisphere involvement, relative mismatch volume, or time from symptom onset to MR imaging between patients with good-versus-poor outcome. Median admission NIHSS score was significantly higher (16 versus $7, P<.0001$ ), and mean DWI, MTT, Tmax, and absolute mismatch volumes were significantly larger (63.4 versus 16.3, 189.9 versus $80.6,217.3$ versus 89.5 , and 127.1 versus $64.9 \mathrm{~mL}$, respectively; all $P<.0001$ ) in patients with poor outcome. More patients with poor outcome than with good outcome had proximal occlusions (ICA or MCA M1) versus MCA M2/M3 or no identifiable occlusion $(P<$ $.0001)$. Sixty-one patients $(49.6 \%)$ received IV-tPA, and 25 $(20.3 \%)$ received IA thrombolysis. Thirty-seven $(30.1 \%)$ patients did not receive IV or IA therapy.

\section{Validation of the Previously Described Combined Thresholds to Predict Outcome in Acute Ischemic Stroke} When applying the previously reported thresholds of DWI $>70$ $\mathrm{mL}$ or NIHSS score $>20$ to predict poor outcome and $\mathrm{MTT}<50$ $\mathrm{mL}$ or NIHSS score $<8$ to predict good outcome to our patient cohort, we found the following: Combining DWI with MTT (or Tmax) led to $88.3 \%$ (or $87.9 \%$ ) PPV, with $48.8 \%$ (or $47.2 \%$ ) prognostic yield (Table 2 ). For NIHSS score $<8$ or $>20$, the PPV was $94.3 \%$ with a $43.1 \%$ yield. Combining the NIHSS score with imaging thresholds improved prognostic yield to $65.0 \%$, higher than that for NIHSS or DWI and MTT alone $(P<.01)$, with a high PPV (88.8\%, 71/80 patients). Figure 1 shows scatterplots of all patients dichotomized into good-versus-poor outcome for the different thresholds. Receiver operating characteristic analysis revealed $95.6 \%(87.6 \%-99.1 \%)$ specificity and $36.4 \%$ $(23.8 \%-50.4 \%)$ sensitivity to predict poor outcome for DWI volume $>70 \mathrm{~mL}$. For MTT volume $<50 \mathrm{~mL}, 92.7 \%(82.4 \%-$ $98.0 \%)$ specificity and $50.0 \%(37.6 \%-62.4 \%)$ sensitivity to predict good outcome were found. For Tmax volume $<50 \mathrm{~mL}$, $92.7 \%(82.4 \%-98.0 \%)$ specificity and $46.2 \%(33.7 \%-59.0 \%)$ sensitivity to predict good outcome were found. AUCs were 
Table 1: Baseline clinical and imaging characteristics, treatment, and predictors of 3-month mRS

\begin{tabular}{|c|c|c|c|c|}
\hline & All Patients $(n=123)$ & $\begin{array}{c}\text { mRS 0-2 } \\
(n=68)\end{array}$ & $\begin{array}{c}\text { mRS 3-6 } \\
(n=55)\end{array}$ & $P$ Value \\
\hline Age (yr) & $69.8 \pm 15.9$ & $68.9 \pm 16.9$ & $70.8 \pm 14.7$ & $.727^{\mathrm{a}}$ \\
\hline Baseline NIHSS score & $11(6-16)$ & $7(5-11.5)$ & $16(14-20.75)$ & $<.0001^{\mathrm{a}}$ \\
\hline Female sex & $55(44.7 \%)$ & $31(45.6 \%)$ & $24(43.6 \%)$ & $.973^{b}$ \\
\hline Right hemisphere & $74(47.2 \%)$ & $34(50.0 \%)$ & $24(43.6 \%)$ & $.602^{\mathrm{b}}$ \\
\hline Time to MRI & $3: 57 \pm 1: 26$ & $3: 45 \pm 1: 29$ & $4: 12 \pm 1: 20$ & $.085^{c}$ \\
\hline DWI volume (mL) & $37.4 \pm 47.7$ & $16.3 \pm 20.1$ & $63.4 \pm 58.2$ & $<.0001^{\mathrm{a}}$ \\
\hline MTT volume (mL) & $129.5 \pm 101.3$ & $80.6 \pm 78.3$ & $189.9 \pm 94.1$ & $<.0001^{\mathrm{a}}$ \\
\hline Tmax volume (mL) & $148.1 \pm 114.9$ & $89.5 \pm 86.5$ & $217.3 \pm 106.0$ & $<.0001^{\mathrm{a}}$ \\
\hline Absolute mismatch (mL) & $92.7 \pm 81.4$ & $64.9 \pm 69.1$ & $127.1 \pm 82.9$ & $<.0001^{\mathrm{a}}$ \\
\hline Relative mismatch & $8.7 \pm 21.6$ & $10.9 \pm 27.8$ & $6.0 \pm 8.8$ & $.541^{\mathrm{a}}$ \\
\hline \multirow[t]{3}{*}{ Reperfusion therapy } & None: 37 (30.1\%) & $22(32.4 \%)$ & $15(25.4 \%)$ & \\
\hline & IV-tPA: 61 (49.6\%) & $36(52.9 \%)$ & $25(45.5 \%)$ & $.200^{\mathrm{b}}$ \\
\hline & IAT \pm IV-tPA: 25 (20.3\%) & $10(14.7 \%)$ & $15(27.3 \%)$ & \\
\hline \multirow[t]{4}{*}{ Occlusion level } & ICA: 24 (19.5\%) & $6(8.8 \%)$ & $18(32.7 \%)$ & \\
\hline & MCA M1: 39 (31.7\%) & 15 (22.1\%) & $24(43.6 \%)$ & $<.0001^{\mathrm{b}}$ \\
\hline & MCA M2/3: 36 (29.3\%) & $24(35.3 \%)$ & $12(21.8 \%)$ & \\
\hline & None: 24 (19.5\%) & $23(33.8 \%)$ & $1(1.8 \%)$ & \\
\hline
\end{tabular}

Note:-IAT indicates intra-arterial thrombolysis.

${ }^{a}$ Mann-Whitney $U$ test.

${ }^{b} \chi^{2}$ test.

c Student $t$ test.

Table 2: Validation of the previously described model combining clinical and imaging thresholds to predict outcome in acute ischemic stroke

\begin{tabular}{lcc}
\hline \multicolumn{1}{c}{ Threshold } & $\begin{array}{c}\text { Positive Predictive } \\
\text { Value (\%) }\end{array}$ & $\begin{array}{c}\text { Prognostic } \\
\text { Yield (\%) }\end{array}$ \\
\hline $\begin{array}{l}\text { Poor outcome } \\
\text { DWI }>70 \mathrm{~mL}\end{array}$ & $86.4^{\mathrm{a}}$ & 17.9 \\
NIHSS score $>20$ & $100.0^{\mathrm{a}}$ & 11.4 \\
Good outcome & & \\
MTT $<0 \mathrm{~mL}$ & $89.5^{\mathrm{a}}$ & 30.9 \\
Tmax $<50 \mathrm{~mL}$ & $88.9^{\mathrm{a}}$ & 29.3 \\
NIHSS score $<8$ & $92.3^{\mathrm{a}}$ & 31.7 \\
Combined clinical thresholds (NIHSS score) & $94.3^{\mathrm{a}}$ & 43.1 \\
Combined imaging thresholds (DWI + MTT) & $88.3^{\mathrm{a}}$ & 48.8 \\
Combined imaging thresholds (DWI + Tmax $>6$ seconds) & $87.9^{\mathrm{a}}$ & 47.2 \\
Combined clinical and imaging thresholds & $88.8^{\mathrm{a}}$ & $65.0^{\mathrm{b}}$ \\
\hline
\end{tabular}

${ }^{a}$ No significant difference in PPV between any of the applied thresholds.

b Significantly increased over use of single parameters $(P<.0001)$ and combined clinical or imaging thresholds $(P=.01)$.

$0.802 \pm 0.04$ for DWI, $0.816 \pm 0.039$ for MTT, and $0.819 \pm$ 0.039 for Tmax (all $P<.0001$ ).

\section{Intravenous or Intra-Arterial Reperfusion Therapy Affects Clinical and Imaging Thresholds}

Stratification by reperfusion therapy (no IV/IA therapy versus IV-tPA alone or IA recanalization \pm preceding IV-tPA) resulted in similar NIHSS scores $(11.0 \pm 7.9$ versus $12.5 \pm 5.8, P=.23)$ as well as DWI $(44.5 \pm 55.0$ versus $35.1 \pm 44.4 \mathrm{~mL}, P=.70)$, MTT $(110.3 \pm 101.8$ versus $137.8 \pm 100.6 \mathrm{~mL}, P=.17)$, and Tmax mean lesion volumes $(125.0 \pm 108.2$ versus $157.6 \pm 116.9 \mathrm{~mL}$, $P=.16)$. However, significant differences in receiver operating characteristic curves and DWI and NIHSS score thresholds for poor outcome were found. Setting specificity to $95 \%$ resulted in optimal thresholds of $>74.8 \mathrm{~mL}$ for DWI and $>19$ for the NIHSS score. For untreated patients, optimal thresholds were lower: $>20.5 \mathrm{~mL}$ for DWI and $>15$ for the NIHSS score. The AUC was greater for DWI in untreated-versus-treated patients $(0.935$ versus $0.724, P=.011$ ). Prognostic yield using DWI was higher for untreated-versus-treated patients (42.3\%-35.1\% versus $19.5 \%-10.4 \%$, Table 3). The mean DWI volume was lower in untreated-versus-treated patients with good outcome $(9.2 \pm 1.7$ versus $19.8 \pm 3.4 \mathrm{~mL}, P=.033)$, and the median NIHSS score ( 5 versus $9.5, P=$ .004 , Fig 2A) was also lower. Probability plots for poor outcome based on logistic regression show a flatter curve for treated patients, which plateaus at approximately 100-mL DWI infarct volume. In contrast, the curve plateaus at approximately 50 $\mathrm{mL}$ for untreated patients (Fig $2 B$ ).

\section{Side of Involvement Affects Clinical and Imaging Thresholds}

The DWI threshold set at 95\% specificity for poor outcome was lower with leftsided $(>51.8 \mathrm{~mL})$ than right-sided strokes $(>98.5 \mathrm{~mL})$, and NIHSS score thresholds were higher $(<9$ or $>20$ versus $<5$ or $>16$ ) when the left hemisphere was affected. Prognostic yield was higher with strokes involving the left side: For DWI, the yield was $40.0 \%-16.9 \%$ versus $20.7 \%-10.4 \%$, and for NIHSS score, the yield was $66.2 \%-38.5 \%$ versus $29.3 \%$ $1.7 \%$ (Table 4). Furthermore, the AUC was higher for DWI with left-sided strokes ( 0.875 versus $0.706, P=.043$ ), and there was a trend toward a higher AUC with the NIHSS score (0.911 versus $0.808, P=.077)$. The mean DWI volume in patients with good outcome was smaller in left- versus right-sided strokes (Fig 3A). A probability plot for poor outcome demonstrates better predictability for left-sided strokes (Fig 3B). Scatterplots for good-versus-poor outcome show that with left-sided strokes, no patient with an NIHSS score of $>20$ (or $<8$ ) had good (or poor) outcome, while for right-sided strokes, no patients with NIHSS scores of $>17$ had good outcome, and some patients with NIHSS scores of $<8$ had poor outcome (Fig $3 B$ ). PPV of our model was $97.4 \%$ for left-sided strokes, and $82.1 \%$ for right-sided strokes $(P=.028)$.

\section{Patients in Whom Imaging or Clinical Thresholds Predicted Clinical Outcome Incorrectly}

Three patients had poor outcomes despite an admission NIHSS score of $<8$. All 3 had right-sided MCA strokes, and 2 had critical ICA stenoses with substantial infarct growth after initial evaluation (On-line Table and On-line Figure, patient 3). Three patients had MTT abnormalities of $<50 \mathrm{~mL}$, but poor outcome. All $3 \mathrm{had}$ significant comorbidities and/or complicated recoveries. Also, patient 5 (On-line Figure) had an infarct in the precentral gyrus, a highly eloquent region strongly represented on the mRS score. Three patients had DWI abnormalities of $>70 \mathrm{~mL}$ but 
DWI

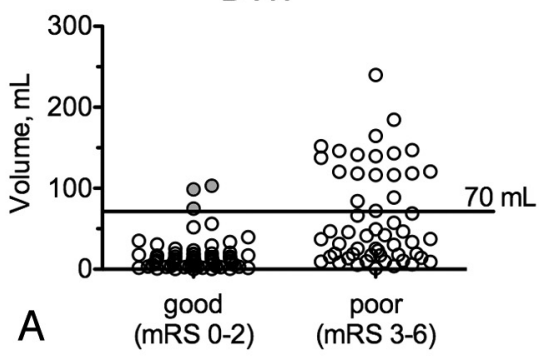

MTT

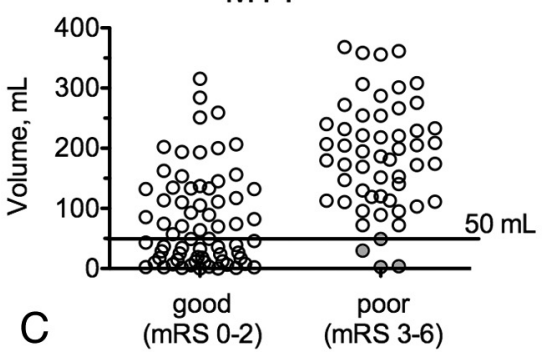

NIHSSS

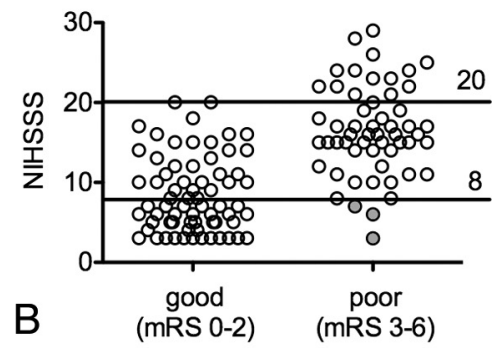

Tmax

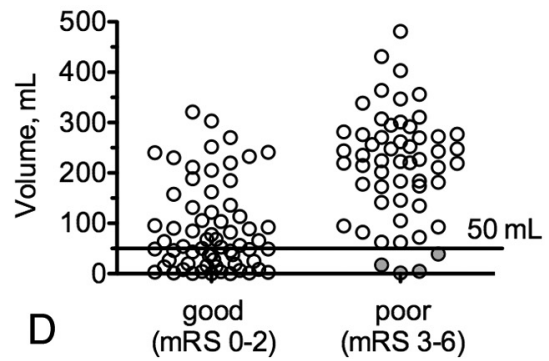

FIG 1. Baseline imaging volumes and NIHSS scores of patients with good (mRS $0-2$ ) and poor (mRS 3-6) clinical outcome at 90 days after stroke. Horizontal lines indicate the thresholds used to predict outcome with DWI $(A)(>70 \mathrm{~mL}$ MTT predicts poor outcome), NIHSS score $(B)(<8$ or $>20$ predicts good or poor outcome, respectively), MTT (C) $(<50 \mathrm{~mL}$ predicts good outcome), and Tmax $(D)(<50 \mathrm{~mL}$ predicts good outcome). The filled circles mark patients for whom prediction was incorrect.

Table 3: Differences of clinical and imaging thresholds in patients receiving thrombolysis versus untreated patients

\begin{tabular}{|c|c|c|c|}
\hline & $\begin{array}{c}\text { No Reperfusion } \\
\text { Therapy }\end{array}$ & $\begin{array}{l}\text { IV and/or } \\
\text { IA Therapy }\end{array}$ & $P$ Value \\
\hline \multicolumn{4}{|l|}{ DWI } \\
\hline $\mathrm{AUC} \pm \mathrm{SE}$ for predicting poor outcome & $0.935 \pm 0.06$ & $0.724 \pm 0.05$ & .011 \\
\hline $90 \%$ Specificity for poor outcome (prognostic yield) $(\mathrm{mL})$ & $>19.7(42.3 \%)$ & $>51.8(19.5 \%)$ & $.016^{\mathrm{a}}$ \\
\hline \multirow[t]{2}{*}{ 95\% Specificity for poor outcome (prognostic yield) $(\mathrm{mL})$} & $>20.5(40.5 \%)$ & $>74.8(16.1 \%)$ & $.007^{\mathrm{a}}$ \\
\hline & $>30.6(35.1 \%)$ & $>103.1(10.4 \%)$ & $.003^{\mathrm{a}}$ \\
\hline \multicolumn{4}{|l|}{ NIHSS score } \\
\hline $\mathrm{AUC} \pm \mathrm{SE}$ for predicting poor outcome & $0.903 \pm 0.06$ & $0.827 \pm 0.04$ & .314 \\
\hline $90 \%$ Specificity for poor outcome (prognostic yield) & $>12(37.8 \%)$ & $>16(23.0 \%)$ & $.142^{\mathrm{a}}$ \\
\hline $95 \%$ Specificity for poor outcome (prognostic yield) & $>15(27.0 \%)$ & $>19(14.9 \%)$ & $.182^{\mathrm{a}}$ \\
\hline $100 \%$ Specificity for poor outcome (prognostic yield) & $>17(16.2 \%)$ & $>20(9.2 \%)$ & $.414^{\mathrm{a}}$ \\
\hline
\end{tabular}

a $P$ value for comparison among prognostic yields.
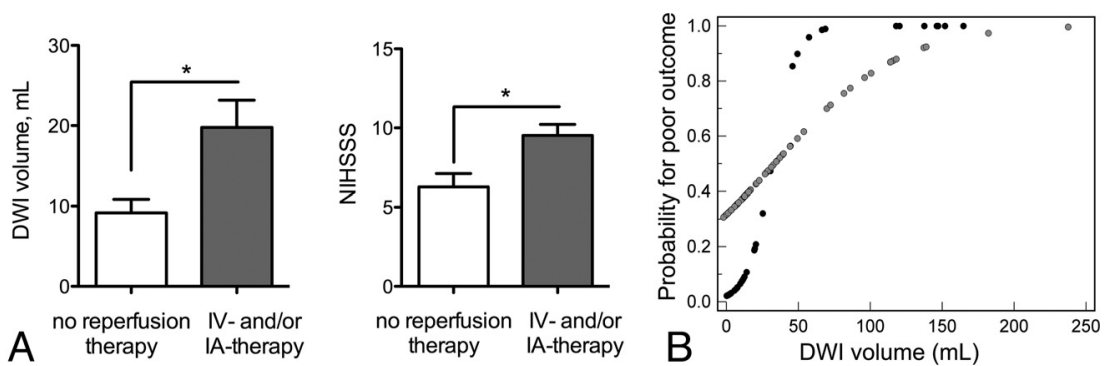

FIG 2. Baseline infarct volumes, clinical status, and probability for poor outcome in treatedversus-untreated patients. A, Mean DWI volume (left) and NIHSS score (right) in patients with good outcome stratified by treatment; asterisks indicate a significant difference. $B$, Probability (determined by logistic regression) for poor outcome versus DWI plots between untreated and treated patients (black circles represent untreated; gray circles, treated).

good outcome. These were relatively young patients $(26-45$ years of age) who all underwent reperfusion therapy (twothirds had received IA thrombolysis and had uncomplicated

recoveries without significant infarct extension). Two also had right-sided strokes.

\section{DISCUSSION}

We have validated our previously described predictive thresholds of DWI $>$ $70 \mathrm{~mL}$ or NIHSS $>20$ for poor outcome of patients with acute ischemic stroke and of MTT $<50 \mathrm{~mL}$ or NIHSS score $<8$ for good outcome, with a high PPV in this much larger, entirely independent cohort. In the $65 \%$ of patients who met at least 1 of these criteria, outcome was predictable with an $89 \%$ PPV. Indeed, we identified only 9 of 123 patients in whom an imaging or clinical threshold predicted clinical outcome incorrectly. Furthermore, we extended our previous results by comparing treated (with IV or IA therapy) versus untreated patients and by comparing right- versus left-sided strokes.

Factors involved in patient selection for reperfusion therapy are nuanced and are currently based on the time from onset and neurologic deficit. Advanced imaging may help to further refine these parameters and more appropriately select patients for intervention. Currently, the most frequently proposed advanced MR imaging method to select patients for intravenous reperfusion therapy is the mismatch between DWI and certain PWI lesion sizes. ${ }^{4}$ Although some evidence supports this strategy, ${ }^{14,15}$ it has not conclusively predicted a favorable treatment response. ${ }^{5-8}$ In addition, relative mismatch measurements fail to account for the absolute sizes of the infarct core and abnormally perfused areas. ${ }^{7}$ In the Echoplanar Imaging Thrombolytic Evaluation Trial (EPITHET), absolute Tmax and DWI lesion volumes influenced the response to reperfusion, but relative mismatch did not. ${ }^{16}$ Finally, DWI/PWI mismatch does not reflect the patient's clinical status. Clinical status, as measured by the NIHSS score, is an important independent predictor of outcome ${ }^{11}$ and neurologic worsening. ${ }^{17}$ In general, in our predictive model, patients with DWI lesions of $>70 \mathrm{~mL}$ or an NIHSS score of $>20$ have a high likelihood of poor outcome, and reperfusion therapy in these patients may offer minimal or no clinical benefit. Patients with MTT lesions of $<50 \mathrm{~mL}$ or an NIHSS score of $<8$ have a high likelihood of good outcome. Aggressive treatments may unnecessarily expose this group to the risks of invasive 


\begin{tabular}{|c|c|c|c|}
\hline & Right Side & Left Side & $P$ Value \\
\hline \multicolumn{4}{|l|}{ DWI } \\
\hline$A U C \pm S E$ & $0.706 \pm 0.07$ & $0.875 \pm 0.04$ & .043 \\
\hline $90 \%$ Specificity for poor outcome (prognostic yield) $(\mathrm{mL})$ & $>39.6(20.7 \%)$ & $>23.4(40.0 \%)$ & $.034^{\mathrm{a}}$ \\
\hline $95 \%$ Specificity for poor outcome (prognostic yield) (mL) & $>98.5(12.1 \%)$ & $>51.8(26.2 \%)$ & $.082^{\mathrm{a}}$ \\
\hline $100 \%$ Specificity for poor outcome (prognostic yield) $(\mathrm{mL})$ & $>103.1(10.4 \%)$ & $>74.8(16.9 \%)$ & $.435^{\mathrm{a}}$ \\
\hline \multicolumn{4}{|l|}{ NIHSS score } \\
\hline $\mathrm{AUC} \pm \mathrm{SE}$ & $0.808 \pm 0.06$ & $0.911 \pm 0.03$ & .077 \\
\hline 90\% Specificity for poor or good outcome (prognostic yield) & $>15$ or $<6(29.3 \%)$ & $>17$ or $<10(66.2 \%)$ & $<.001^{\mathrm{a}}$ \\
\hline $95 \%$ Specificity for poor or good outcome (prognostic yield) & $>16$ or $<5(17.2 \%)$ & $>20$ or $<9(49.2 \%)$ & $<.001^{\mathrm{a}}$ \\
\hline $100 \%$ Specificity for poor or good outcome (prognostic yield) & $>16$ or $<3(1.7 \%)$ & $>20$ or $<7(38.5 \%)$ & $<.001^{\mathrm{a}}$ \\
\hline
\end{tabular}

a $P$ value for comparison between prognostic yields.

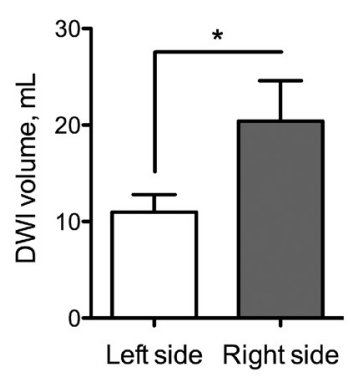

A

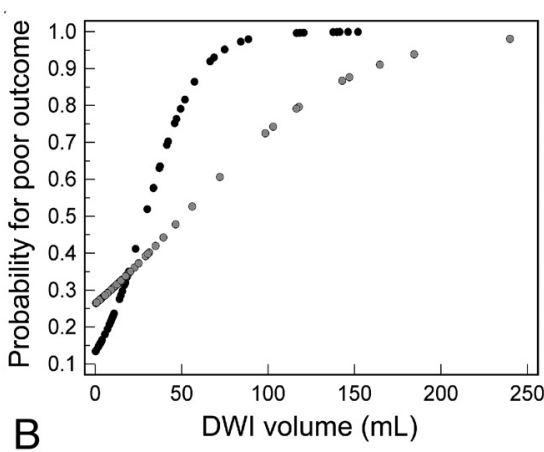

B

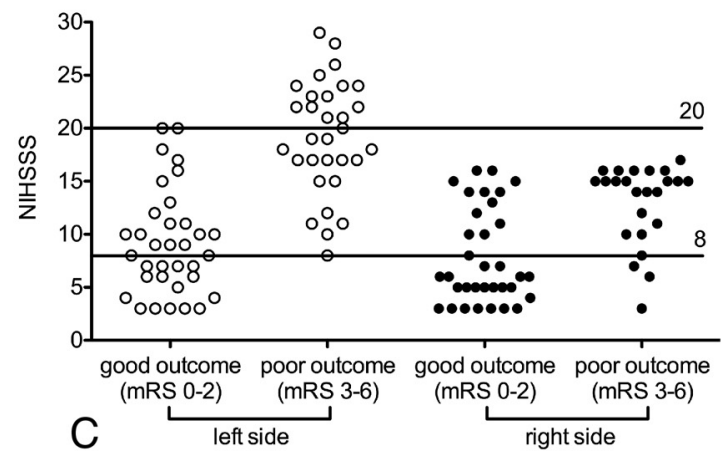

FIG 3. Baseline infarct volumes, probability for poor outcome, and NIHSS score thresholds in left- versus right-sided strokes. $A$, Mean DWI volume in patients with good outcome stratified by side of involvement. Asterisks indicate a significant difference. $B$, Probability (determined by logistic regression) for poor outcome versus DWI plots between left- and right-sided strokes (left $=$ black circles, right $=$ gray circles). $C$, NIHSS score scatterplots and thresholds stratified by side of involvement.

reperfusion therapy. The outcomes of the remaining $35 \%$ of patients, those with DWI volume of $<70 \mathrm{~mL}$, MTT volume of $>50$ $\mathrm{mL}$, and an NIHSS score of $>8$ and $<20$, could not be predicted accurately by initial clinical and imaging assessments; this finding suggests that these patients may represent a group toward whom early invasive therapy may be directed.

We hypothesized that patients who received IV thrombolytic and/or IA recanalization therapy would have higher DWI and NIHSS score thresholds for poor outcome than untreated patients. Indeed, the $100 \%$ specific DWI threshold for poor outcome was $103 \mathrm{~mL}$ in treated compared with $31 \mathrm{~mL}$ in untreated patients, and the $100 \%$ specific NIHSS score thresholds were similarly 20 versus 17, respectively, suggesting that treated patients may tolerate larger baseline infarcts. This suggestion is also supported by significantly larger DWI lesions and higher NIHSS scores in treatedversus-untreated patients with good outcome. An explanation for this finding might be that in patients treated with reperfusion therapy, infarcts grow less than in untreated patients. When predicting good outcome with the NIHSS score, MTT, or Tmax, we did not find conclusive evidence for differences by treatment. We did not further stratify patients by different treatment strategies and recanalization status (eg, IV-versus-IA thrombolysis) because recanalization status was unknown in the 61 patients treated with IV therapy only and our sample size was too small.

Because the dominant (usually left) hemisphere has important functions represented in the mRS, we hypothesized that patients with right-sided strokes would tolerate larger DWI lesions and that our model would be less accurate. Indeed, the AUCs were significantly higher for DWI ( 0.88 versus $0.71, P=.04)$ with left- sided strokes. The difference in AUC for the NIHSS score in leftversus right-sided strokes also approached significance ( 0.91 versus $0.81, P=.08)$. Moreover, patients with right-sided strokes could tolerate larger DWI lesions (100\% specific DWI volume for poor outcome $=103$ versus $75 \mathrm{~mL}$ ), and on average, patients with right-sided strokes and good outcome had significantly larger DWI lesions (20 versus $10 \mathrm{~mL}, P<.05$ ). No patient with an infarct affecting the right hemisphere had an NIHSS score of $>17$, making outcome prediction in these patients less reliable.

In the 9 patients for whom the above clinical or imaging thresholds predicted outcome incorrectly, we identified 4 scenarios: 1) Young patients undergoing reperfusion may do well with DWI lesion volumes of $>70 \mathrm{~mL}$, in accordance with studies suggesting better outcome in patients younger than 40 years of age. ${ }^{18}$ 2) Factors delaying rehabilitation or causing infarct growth, such as comorbidities, complicated recovery, and irregular clinical course, might lead to poor outcome despite MTT lesion volumes of $<50 \mathrm{~mL}$. 3) Infarcts in areas affecting functions highly represented in the mRS score (eg, motor function in the precentral gyrus) may result in poor outcome despite small MTT lesion volumes. This possibility is underscored by a recent study, in which infarct location on MR imaging correlated highly with outcome. ${ }^{19}$ 4) NIHSS score and mRS are strongly biased toward dominant (usually left) hemisphere functions. All patients with false prediction based on an NIHSS score of $<8$ had right-sided infarcts, and two-thirds of patients with infarct volumes of $>70 \mathrm{~mL}$ and good outcome had right-sided strokes. The challenges to prediction represented by these variables are important to keep in mind when evaluating patients for therapy. 
The limitations of our investigation are the consequences of the retrospective design and have been described elsewhere. ${ }^{12}$ Briefly, we could not obtain data such as reperfusion status, final infarct volumes, and clinical status at day 30 for most patients. There were variable treatments and variable times from stroke onset to imaging and from imaging to follow-up clinical assessment. Potential selection bias was related to using MR imaging, the decision to offer thrombolysis, and our technical approach to performing IA therapy. While assessment of Tmax maps was based on a 6-second threshold, DWI and MTT lesions were outlined by visual estimation. Thresholds have been proposed to increase reliability, but most studies demonstrating a clinical benefit from perfusion imaging-based patient selection have used visual estimation. ${ }^{14,15}$ No thresholding software is universally accepted, and our data suggest that both methods can be used in our model with similar yield and PPV. Finally, our method for measuring DWI and PWI lesion volumes is too time-consuming for routine clinical use. The faster ABC/2 volume estimation method based on 3 orthogonal measurements, easily obtained on an MR imaging scanner console, could be a feasible substitute. ${ }^{20}$

\section{CONCLUSIONS}

The previously proposed predictive model combining DWI, MTT, and NIHSS score thresholds is validated in a much larger patient cohort. For acute ischemic stroke, thresholds applied to acute DWI and MTT lesion volumes and NIHSS scores can be used to predict good and poor clinical outcomes with a high PPV. Our prediction model shows higher PPV in left-sided strokes. Younger patients, those treated with IV and/or IA therapy, and those with right-sided strokes may have good outcomes despite larger DWI infarcts. Those treated with reperfusion therapy may also tolerate a higher NIHSS score. These variables, alone and in combination, may help guide decisions related to patient selection for therapy. Patients who do not meet these thresholds have variable outcomes and may represent a target population for more aggressive revascularization approaches.

Disclosures: Joshua A. Hirsch-UNRELATED: Consultancy: CareFusion, Comments: In $>24$ months and $<36$ months, I received compensation related to vertebral augmentation; Royalties: CareFusion (as above); Stock/Stock Options: Intratech, Comments: development-stage company for interventional stroke products. Lee $\mathrm{H}$. Schwamm-UNRELATED: Consultancy: Lundbeck, Comments: Member of data safety monitoring board for Desmoteplase In Acute Ischemic Stroke trial threefourths trials of desmoteplase; Grants/Grants Pending: National Institute of Neurological Disorders and Stroke/Genentech, ${ }^{\star}$ Comments: Genentech provides drugs free of charge and some supplemental site payments for the MR WITNESS trial of extended window thrombolysis (Dr Schwamm is the Principal Investigator). Ona Wu-RELATED: Grant: National Institutes of Health,* Comments: R01NS059775, R01NS063925, P50NS051343, P41EB015896; UNRELATED: Consultancy: Penumbra, Comments: provided consultancy regarding digital imaging and communications in medicine servers and clients; Grants/Grants Pending: Genentech, ${ }^{*}$ Comments: Genentech has provided funds to MR WITNESS to help per-subject reimbursement for each site; OTHER RELATIONSHIPS: I have a patent on "Delay-Compensated Calculation of Tissue Blood Flow," US Patent 7,512,435, March 31, 2009, and the patent has been licensed to GE Healthcare, Siemens, Imaging Biometric, and Olea Medical. AlbertJ. YooUNRELATED: Grants/Grants Pending: Penumbra, ${ }^{*}$ Comments: core imaging lab. *Money paid to the institution.

\section{REFERENCES}

1. Tissue plasminogen activator for acute ischemic stroke: the National Institute of Neurological Disorders and Stroke rt-PA Stroke Study Group. N Engl J Med 1995;333:1581-87
2. Hacke W, Kaste M, Bluhmki E, et al. Thrombolysis with alteplase 3 to 4.5 hours after acute ischemic stroke. N Engl J Med 2008;359: 1317-29

3. Cocho D, Belvis R, Marti-Fabregas J, et al. Reasons for exclusion from thrombolytic therapy following acute ischemic stroke. $\mathrm{Neu}$ rology 2005;64:719-20

4. Schlaug G, Benfield A, Baird AE, et al. The ischemic penumbra: operationally defined by diffusion and perfusion MRI. Neurology 1999;53:1528-37

5. Davis SM, Donnan GA, Parsons MW, et al. Effects of alteplase beyond $3 \mathrm{~h}$ after stroke in the Echoplanar Imaging Thrombolytic Evaluation Trial (EPITHET): a placebo-controlled randomised trial. Lancet Neurol 2008;7:299-309

6. Mishra NK, Albers GW, Davis SM, et al. Mismatch-based delayed thrombolysis: a meta-analysis. Stroke 2010;41:e25-33

7. Hacke W, Furlan AJ, Al-Rawi Y, et al. Intravenous desmoteplase in patients with acute ischaemic stroke selected by MRI perfusiondiffusion weighted imaging or perfusion CT (DIAS-2): a prospective, randomised, double-blind, placebo-controlled study. Lancet Neurol 2009;8:141-50

8. Kidwell CS, Jahan R, Gornbein J, et al. A trial of imaging selection and endovascular treatment for ischemic stroke. $N$ Engl J Med 2013;368:914-23

9. Sanak D, Nosal V, Horak D, et al. Impact of diffusion-weighted MRI-measured initial cerebral infarction volume on clinical outcome in acute stroke patients with middle cerebral artery occlusion treated by thrombolysis. Neuroradiology 2006;48:632-39

10. Yoo AJ, Verduzco LA, Schaefer PW, et al. MRI-based selection for intra-arterial stroke therapy: value of pretreatment diffusionweighted imaging lesion volume in selecting patients with acute stroke who will benefit from early recanalization. Stroke 2009; 40:2046-54

11. Sato S, Toyoda K, Uehara T, et al. Baseline NIH Stroke Scale score predicting outcome in anterior and posterior circulation strokes. Neurology 2008;70:2371-77

12. Yoo AJ, Barak ER, Copen WA, et al. Combining acute diffusionweighted imaging and mean transmit time lesion volumes with $\mathrm{Na}$ tional Institutes of Health Stroke Scale score improves the prediction of acute stroke outcome. Stroke 2010;41:1728-35

13. Wu O, Østergaard L, Weisskoff RM, et al. Tracer arrival timinginsensitive technique for estimating flow in MR perfusionweighted imaging using singular value decomposition with a blockcirculant deconvolution matrix. Magn Reson Med 2003;50:164-74

14. Furlan AJ, Eyding D, Albers GW, et al. Dose Escalation of Desmoteplase for Acute Ischemic Stroke (DEDAS): evidence of safety and efficacy 3 to 9 hours after stroke onset. Stroke 2006;37:1227-31

15. Hacke W, Albers G, Al-Rawi Y, et al. The Desmoteplase in Acute Ischemic Stroke Trial (DIAS): a phase II MRI-based 9-hour window acute stroke thrombolysis trial with intravenous desmoteplase. Stroke 2005;36:66-73

16. Parsons MW, Christensen S, McElduff P, et al. Pretreatment diffusion- and perfusion-MR lesion volumes have a crucial influence on clinical response to stroke thrombolysis. J Cereb Blood Flow Metab 2010;30:1214-25

17. DeGraba TJ, Hallenbeck JM, Pettigrew KD, et al. Progression in acute stroke: value of the initial NIH stroke scale score on patient stratification in future trials. Stroke 1999;30:1208-12

18. Galldiks N, Zaro-Weber O, Dohmen C, et al. Systemic thrombolysis with rt-PA in patients under $\mathbf{4 0}$ years of age: a subgroup analysis of the Cologne Stroke Experience. Cerebrovasc Dis 2010;30:514-18

19. Phan TG, Chen J, Donnan G, et al. Development of a new tool to correlate stroke outcome with infarct topography: a proof-of-concept study. Neuroimage 2010;49:127-33

20. Sims JR, Gharai LR, Schaefer PW, et al. ABC/2 for rapid clinical estimate of infarct, perfusion, and mismatch volumes. Neurology 2009;72:2104-10 